\title{
Ideas para el acceso libre a la información
} de vanguardia

\section{Departamento de Formación y Actualización Académica}

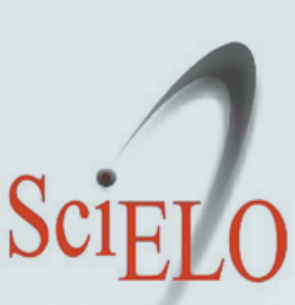

Scientific Electronic Library Online www.scielo.org.mx

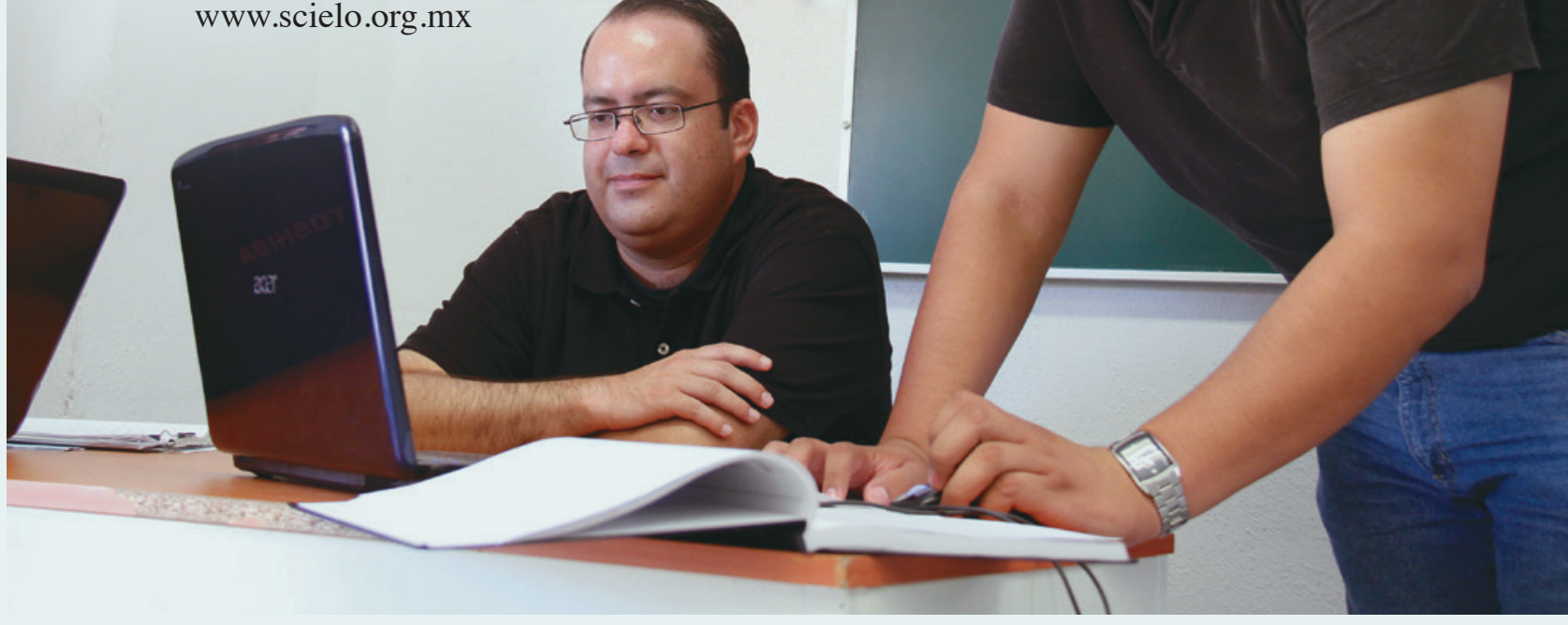

S

iELO (Scientific Electronic Library Online) es una hemeroteca virtual conformada por una red de colecciones de revistas científicas en texto completo y de acceso abierto y gratuito.

El sitio ofrece una hemeroteca virtual con acceso a textos completos de artículos que han sido publicados en una amplia colección de revistas académicas tanto de México como de Argentina, Chile, Bolivia, Brasil, Colombia, Costa Rica, Cuba, España, Paraguay, Perú, Portugal, Sudáfrica, Uruguay y Venezuela. Dicha colección es desarrollada por la Dirección General de Bibliotecas (DGB) de la Universidad Nacional Autónoma de México (UNAM), la cual recibe financiamiento del Consejo Nacional de Ciencia y Tecnología (CONACYT).

A través del portal www.scielo.org, que integra y provee acceso a la red de sitios SciELO (de los países previamente mencionados), se pueden realizar búsquedas simultáneas en todas las colecciones SciELO existentes o en cada una de las mismas. Por ejemplo, para entrar al sitio de SciELO México y realizar búsquedas de revistas, artículos e 
informes, habrá que ingresar la siguiente dirección en su navegador: www. scielo.org.mx.

Las opciones de búsqueda son diversas: para "revistas", se puede realizar mediante "Lista alfabética", "Lista por materia" o "Búsqueda de títulos".

Para la búsqueda de artículos, SciELO ofrece la opción de buscar por autor en su "Índice de autores" o por temática en su "Índice de materias", así como a través de la "Búsqueda de artículos" en el caso de que el texto de interés no pueda localizarse en los índices antes mencionados. Dentro de esta última opción, se podrán apreciar tres recuadros para escribir y hacer una búsqueda más específica. Por ejemplo: en el primer recuadro, se escribe la palabra: "aprendizaje"; para el segundo recuadro se aprecia un menú desplegable con las siguientes palabras: "and", "or" y "and not", esto para que se pueda especificar qué es lo que se desea obtener y qué no en los resultados.

Retomando el ejemplo: para el segundo recuadro, se seleccionará del menú desplegable "and" y a continuación se escribe la palabra "proyectos", señalando así qué artículos sobre "aprendizaje por proyectos" son los de interés. Y finalmente, si no se desea obtener alguna otra clase de resultado, como "aprendizaje basado en problemas", en el tercer recuadro, después de seleccionar del menú desplegable "and not", se redactará la palabra "problemas", descartando así los artículos relacionados con este tipo de aprendizaje.

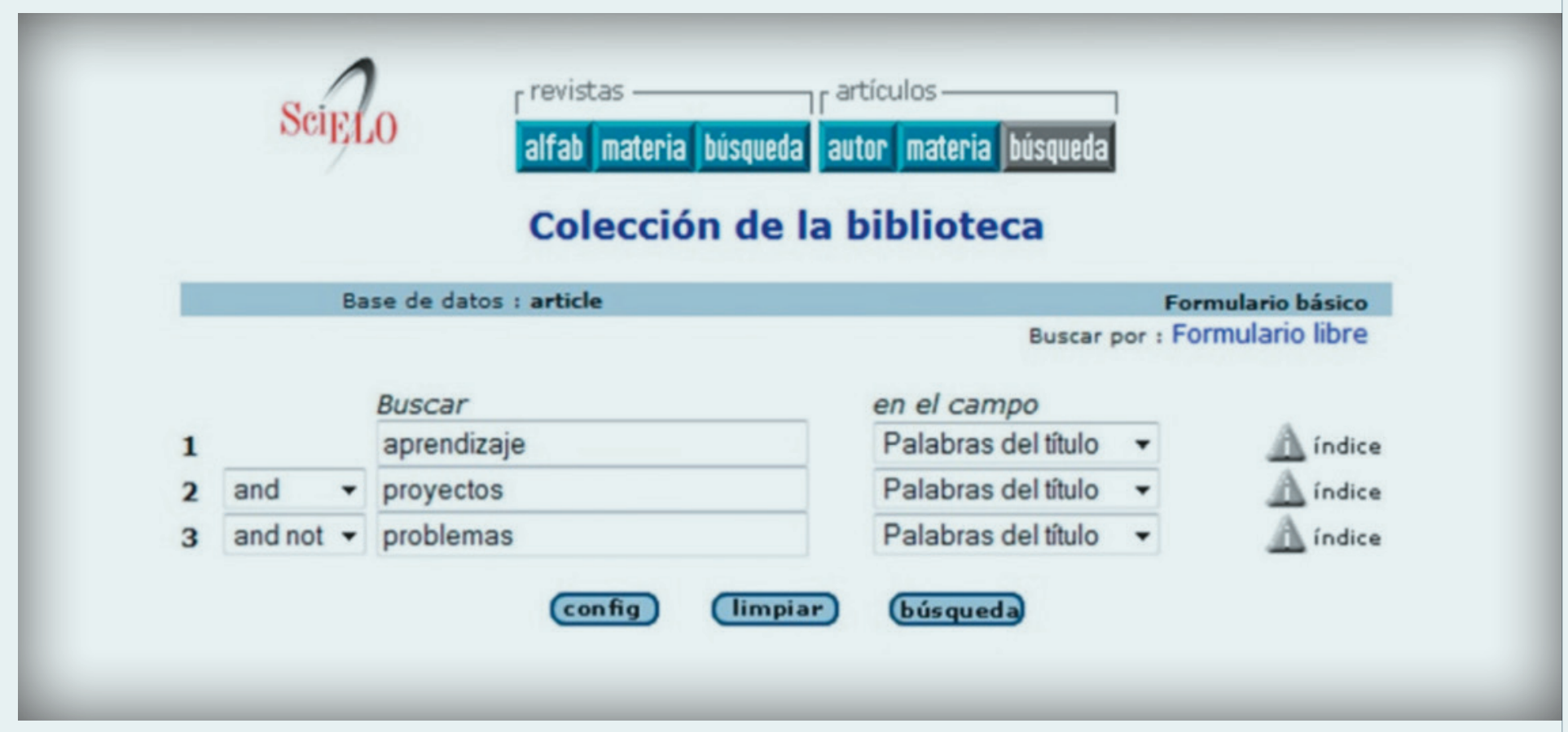

Cabe señalar que delante de cada uno de los recuadros antes mencionados se podrán apreciar otros tres recuadros para seleccionar (en cada uno de ellos a través de un menú desplegable) una búsqueda por "campo", que puede ser: "Palabras del título", "Autor", "Materia", "Resumen" y "Año de publicación".

Por ejemplo, para la palabra "aprendizaje" se seleccionará del menú desplegable: "Palabras del título", y para las palabras "proyectos" y "pro- 
blemas", se seleccionará la misma opción (como el anterior), y se dará clic en el botón "Búsqueda".

Para este ejemplo en específico, la base de datos de SciELO arroja un resultado que proporciona información para citarlo bibliográficamente así como las opciones de descarga del resumen en español e inglés del artículo y el texto completo en el idioma español (en algunos casos también están disponibles en los idiomas portugués y francés). Cabe señalar que en otros casos pudiera ofrecerse la oportunidad de descargar el artículo completo en el idioma inglés.

SciELO es un portal muy útil para realizar búsquedas de textos académicos -en su mayoría completos- por tema, autor, revista o año de publicación. Alberga una gran diversidad de contenidos sobre las diferentes disciplinas del conocimiento, que sin duda serán de gran apoyo en la labor docente.

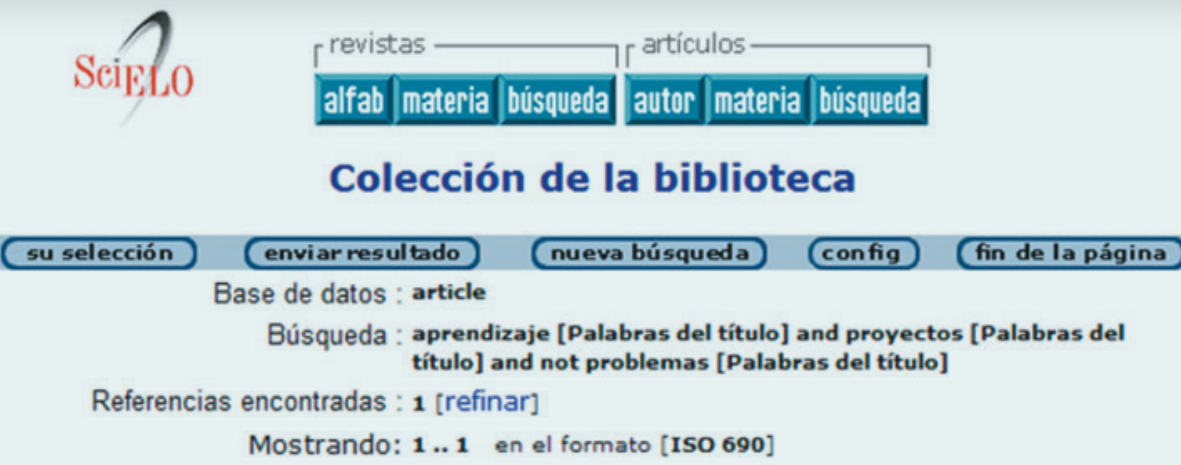

Chacón Corzo, María Auxiliadora, Chacón, Carmen Teresa and Alcedo S., Yesser Antonio Los proyectos de aprendizaje interdisciplinarios en la formación docente. RMIE, Set 2012, vol.17, no.54, p.877-902. ISSN 1405-6666

resumen en español | inglés - texto en español

\section{página 1 de 1}

\begin{tabular}{|l|l|l|} 
& & Buscar \\
\hline $\mathbf{1}$ & & aprendizaje \\
\hline $\mathbf{2}$ & and $\mathbf{*}$ & proyectos \\
\hline $\mathbf{3}$ & and not $\mathbf{}$ & problemas \\
\hline
\end{tabular}

en el campo

\begin{tabular}{|l|l|}
\hline Palabras del título & A índice \\
\hline Palabras del título & -
\end{tabular}

